\title{
GLAD!
}

Revue sur le langage, le genre, les sexualités

03 | 2017

Hétérosexualités

\section{Une genre de nouvelle discrimination chromatique}

A Gen(de)re of New Chromatic Discrimination

\section{R. J. Aeschlimann}

\section{(2) OpenEdition}

Journals

Édition électronique

URL : http://journals.openedition.org/glad/842

DOI : $10.4000 /$ glad. 842

ISSN : 2551-0819

Éditeur

Association GSL

Référence électronique

R. J. Aeschlimann, « Une genre de nouvelle discrimination chromatique », GLAD! [En ligne], 03 | 2017, mis en ligne le 10 décembre 2017, consulté le 20 janvier 2021. URL : http://journals.openedition.org/ glad/842 ; DOI : https://doi.org/10.4000/glad.842

\section{(9) $\odot \Theta \Theta$}

La revue GLAD! est mise à disposition selon les termes de la Licence Creative Commons Attribution -

Pas d'Utilisation Commerciale - Pas de Modification 4.0 International. 


\section{Une genre de nouvelle discrimination chromatique}

Note liminaire écrite du corps traductoral au lectorat

Ci-décrites les diverses gens colorés dont nous mentionnons ci-relaté les caractéristiques phénotypiques sont caractérisés par une multitude de variables structurelles, notamment au niveau de l'anatomie génitale externe, interne, la génétique sexuelle, l'endocrinologie reproductive, l'identité de genre intrapsychique, sociale, son expression permanente ou transitoire, ordinaire ou paroxystique...

Par souci de fluidité de lecture ainsi que pour des raisons évidentes de discrétion du genre, la forme féminine sera utilisée en principe et jusqu'au générique de fin pour la description des "gens en orange" et désignera également tout le continuum des genres grammaticaux.

$\mathrm{Au}$ commencement, ou pour être plus exact , à la première occurrence, Nike lança une nouvelle basket, totalement rouge de la cheville au pied.

La chaussure elle-même était toutàfaitement laide et convenait à l'idiotie d'un prolétariat idoine, mais le géant de la pointure avait fait preuve

d'une virtuosité pénétrante en érigeant un slogan accrocheur: « Tout ce qui est rouge est joli » ${ }^{\dagger}$.

La semelle en soi humait la mycose plantaire avec sa matière plastique bon marché, mais la démarche de la marque était telle que Nike s'en trouva plein aux as ; l'entreprise avait derechef inondé le monde de sa révolution rouge.

Par une belle fin de semaine bâloise, tandis qu'on longeait le Rhin en soirée, il n'y avait que cette nouvelle mode à observer : tou'te's portaient le même rouge. Rouge, il n'y avait que ce mot aux lèvres. « Maman, Papa, j'aimerais les mêmes baskets rouges qu'elle / que lui. » Elle ou lui, en l'occurrence, les portait de préférence avec des pantalons noirs,

« Tout ce qui est rouge est joli - Tout ce qui est nouveau est beau [...] », extrait de "La maladie de Chûchulainn », cité par Nicolas Bouvier en exergue de son « Journal d'Aran » 
troués aux genoux. Chacun_e se trouvait alors moderne|unique, original-e. Grâce aux facilités de l'e_banking et au coût mod|ique de la paire de rouges, les suiv:eur.euse-s n'étaient qu'à une paire de clics du reste des fanfaron,ne,s.

Tou[te]s les product<eur[s]|rice[s] de chaussures créèrent leur version, mais quel(le) que soit la marque $\backslash$ le logo, le rouge demeurait le même. En effet, quelque plus orange ou quelque plus roses que les coloris variassent, aucun+ n'avait plu \pm s envie de les porter.

Il n'était alors pas question de faire sa*son atypique.

Baskets basses, tennis hautes, souliers de vair luisants ou sandales imperméables, tou:t;e,s se devai;en,t d'être de cette teinte de rouge banal|je pour plaire aux filles en leggins troués TALLY WEijL ou'et aux mecs en trainings adidas,

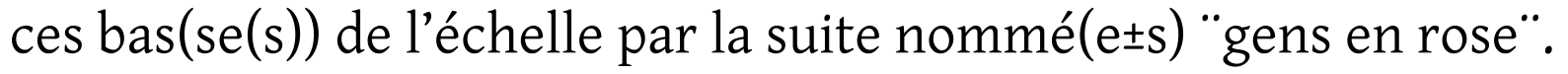

adidas, forte de s'avantage, fit sa maligne en créant la basket rouge à talon, pour les filles travailleuses du bitume et autres bénévoles des rues.

Puma fit son félin et fut fol osant enfin un volte-face à faire rougir les pepsi girls : une basket rouge et bleu pour les prolo des deux sexes, mais attention, pas n'importe comment, rouge à l'extérieur - pour ne pas trop choquer - et bleu à l'intérieur, ce qui donnait au premier abord l'impression que la superhéroïne qui les portait avait alterné deux paires, puisqu'on ne pouvait apercevoir que la face externe (ou interne) du pied droit (ou l'inverse) simultanément avec la face interne (resp. externe) du pied gauche (ou le pléonasme).

Cette version plus entreprenante, plus puissante, plus agressive fit fureur auprès des ironwomen, des spaïderouimines, des Musclaure et des fennes du FCB.

Bientôt toutes les agissantes du monde sportif et acteures de la construction se mirent à préférer les chaussures rouge-bleu et bleu-rouge aux rouges seulement.

Les portières d'hôtel, les chauffeuses de bus, les peintresses en bâtiment, les sumotori professionnelles, les femmes des casernes, les militères, les pilotes de l'ère et toutes les uniformes orange fetishes, ainsi que les pêcheresses et les thons, les entraîneuses de sirènes, les pompières, 
les arbitrères de soccère, les handballeuses, les manouvrières,

les fabriquantes, les faisantes et autres artisanes manuelles.

Et pour les bayadères de sexe féminin, une version à Semelle compensée

fut produite comme alternative progressiste aux hauts talons.

Une erreur de fabricant - voire d'accord - produisit une mutation exceptionnelle.

Suite aux baskets rouge et bleu, il apparut soudain sur le marché des pieds les baskets rouges et bleues. Cette fois-ci, ce n'était plus une illusion d'optique :

la gauche était totalement rouge et la droite totalement bleue.

Méfiant?, presque personne ne voulut acheter cette mésalliance.

Boxeur Des Rues ${ }^{\oplus}$ fut contrainte de doubler sa production afin d'équiper chaque paire d'une gauche bleue ou d'une droite rouge.

Le fabricant essaya de refaire ses lettres de sang bleu

en vendant son surplus de baskets à moitié prix,

afin de faire oublier son nouveau sobriquet ignoble BoxeurE Des RuSes.

Rien n'y fit. Nulle n'acheta la chaussure mutante pour la porter à ses talons.

Les chevalières Jedi préféraient le bicolore tandis que

les fleurs de pavé restaient fidèles au rouge.

Pour que cette nouvelle création s'amorçât enfin, il fallut attendre le nouveau glissement de la marque Freitag, qui confondit la moitié et le double. Sa nouvelle sandale bleu bâche fut erronément commercialisée au double du prix.

Ironiquement, elle fit un malheur auprès de la classe petite-bourgeoise. Les gens féminine et masculine avaient enfin trouvé chaussure à leur prix et toutes les entreprises se mirent à commercialiser un produit synchrome.

La gent masculine en pantalons bleu de Gênes et veste bleu roi et la gent féminine moins bleue quoiqu'y accordée

exhibaient leur conformité à la mode la plus pointue avec leur pointures bleues. Rouge pour exprimer un tempérament sanguin, bleu pour paraître d'un meilleur cru. Et puis bleu, c'était quand même plus viril, plus dominant. 
Puisqu'une marque de sacs avait réussi à prendre pied dans la chaussure, d'autres entreprenantes entreprirent cette entreprise.

Fjällräven Kanken et Herschel y trouvèrent un nouveau fond de commerce. Une étude en doubles aveugles démontra que les achetantEs des baskets rouges et

bleues n'étaient pas les femmes respectivement les hommes, mais les "gens en rose" voire "orange" respectivement les "gens en bleu". La couleur était le symbole du pouvoir d'achat et non celui de l'inégalité des sexes. Ainsi, l'innovation dans la basket grise ne fut pas sa couleur, mais son prix : une hipstérie aussi absurde que la mode des feu de plancher.

Les dessinateurices en art ou en bâtiment, les éternelles étudiantes, les consommateurices et achetantes invértébrées - bref les "gens en gris" se ruèrent sur la chaussure haute en daim gris coûtant l'équivalent de quatre rouges.

Les hipsteres, demeurées les seules à croire que le coût équivalait la qualité. IElles bramaient : "Vous imaginez ces hardes de daimnes teintes en gris... ». Et les vendeureuses de glouglouter : «C'est là_le dindonneau de la farce... »

Yves Saint Laurent, Gucci et d'autres noms de renom ${ }^{\circledR}$ mée boutiquèrent alors des versions beiges, marron et écrues, que l'on s'arracherait alors pour le prix de quatre à dix-neuf nonante-cinq rouges.

Les "gens en beige" (f/h) vouèrent un nouveau culte d'adoration au shopping, comme ils avaient pu le faire auparavant aux décimales de Pi, à la Trinité ou ne serait-ce qu'à Le Fils Unique (homme et femme). « Aristocrates de tous les pays, la chaussure bise est dans le vent!", disait la Parole d'En-Haut. Moult Ayanz et toult les Possédanz avaient enfin trouvé la teinte de beige qui convenait parfaitement à celle des manteaux et sacs à mains de lor épouz ( honpmes oulet femmes)) ou celle des carrosseries et sièges en cuir de lor épouz femmelhomme .

Dans les échoppes, on s'encoublait désormais aux pierres d'achoppement, si bien qu'il fallut ainsi prioriser : «Les enfants (H/F) et les femmes d'abord ! » 
Jamais jusqu'alors dans les hypothèses freudomarxistes les plus tirées par les lacets la chaussure n'avait autant fait l'homme comme la pointure le revenu.

Ainsi, pour résumer (avant que cela ne se compliquât) :

Quiconque $-\partial^{\lambda}+$ - portait des chaussures glacé marron était une personne "en beige"; qui portait des chaussures grises était une personne "en gris" ;

quelconque portait des chaussures bleu Charron était une personne "en bleu"; quelleconque portait des chaussures bleuâtres était une personne "en bleu"; kixesoa*chickcesoir portait des chaussures rouges était une personne "en rose";

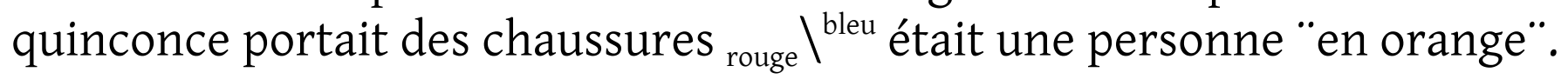

Alors que tot l'élite de la cour $<$ [ho|fe]mme $>$ s'était envolée dans l'empyrée rien qu'en chaussant lors élytres calcanéens d'Hermès,

la partie la plus éclatante et distinguée de la société n'avait pas encore trouvé de quoi briller jusqu'à ce que Prada n'accouchât du talon aiguille doré grâce à l'ingénuité de sages-femmes (hommes dans le sens générique) dépravéєs.

Les $3 . \cdot$ gens en doré $\bullet$ is avaient alors eלux.lles aussi obtenu LEUR couleur qui permettrait désormais aux pauvre $\sim s s e \sim s$ dans la rue de savoir de quel feu d'artifice elles $\equiv$ ils se chauffaient.

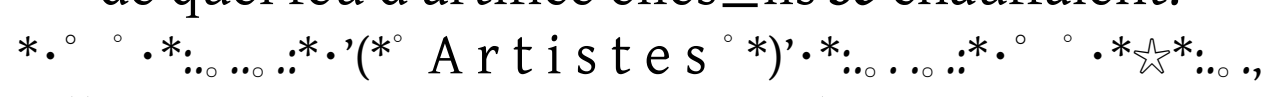

merveilleuses $\delta\left({ }^{*} s \mathrm{t}\right.$ a $\left.\mathrm{r}^{\wedge}\right) \circ \forall^{*} \forall \mathrm{o}\left({ }^{\wedge} \mathrm{s}\right.$ t a r l e t t e $\left.\mathrm{s}^{\wedge *}\right) \delta$ vermeilles, adeptes $\ll$ du botox, siliconés $\nsim$ silliconnasses, bodybuild hé:roÿs-Soleilohé:roïnes-Cryštal de bas talent,

les $\neg$ adeptes $<$ de $\rightarrow$ l'excèse $\leftarrow$ outrepassèrent une fois de plus les limites et ne se contentèrent pas seulement de leurs talons d'or qui coûtaient alors entre quarante et carat-vingt mil fois les baskets rouges, mais ils se mirent à p o u s s e r l'or consommation de dorure $\$$ et de mercure¥ au point que ${ }_{\text {Nous }}$ commissions pour eur $\equiv$ eux d'autres produits éclectiques : plumeaux d'orfraies d'or feint, manteaux de léopardı d'or, argenterie dorée, toisons d'or, cartes d'or, scarabées d'or, lions d'or, boîtes de pan d'or et berlingots. 
La nouvelle tendance des objets (femmes également) dérivé(e)s en doré se popularisa bientôt auprès des "gens en vert".

Les gens en vert, princesses-crapauds et princes-grenouilles, pullulent dans toutes les classes sociales comme le hibou bouboule et la chouette chuinte.

Les hommes en vert et les femmes en vert ne sont pas du genre à être des rats de bibliothèque respectivement des souris de bibliothèque.

Ce sont des êtres originaux et des faînes originelles qui n'ont pas peur de se montrer vêtus et habillées de vert clair, moiré, fluo, et se démarquent par leur dernier cri - ou hululement ultime.

Ces hurluberlus et ces hurluberlüs, les femmes en vert ou les hommes en vert, étaient des m'as-tu-vu et des m'as-tu-vu belles et rebelles et ils-ou-et-elles furent bientôt reconnaissables à leurs grolles vertes, pistache ou vermillon, voire rose bonbons, incarnates, infrarouges parfois.

Nous-même, camouflé voire camouflée en vert et contre tous, sous son arbre à palabre, un auteur-compositeur-sécateur ou une auteure-compositrice-sécateuse observait les passants, les passagères, les géants et les gérantes faires les cent pas à toute pompe.

Et nous observait qu'il n'y avait pas que les chaussures, vertes ou pas, qui faisaient les "gens en vert", mais aussi tous les attributs herméneutiques, plumeaux, manteaux, léopardeaux, lionceaux, caducée vert et tous les noms d'oiseau, d'oiselle, d'oisillon - voire d'oisillonne hirondelle/hirondelle/hirondeau, moineau/moinelle/moinet, flamants verts et vertes flamboyants et flamboyant.

Une étape fondamentale venait d'avoir eu lieu : ce n'était plus la couleur de la chaussure qui permettait de savoir si l'on s'adressait à une entraîneuse "en orange" ou une professionnelle "en doré", mais toute une constellation d'accessoires bariolés et flambant neufs. 
L'œil averti en valait deux : on pouvait désormais être "en bleu" sans être en bleu, voire être une femme "en orange" sans être ni femme ni en rouge ni en bleu.

On reconnaissait alors l'"h hoemme en gris"

à sa casquette de truckeure et non de baseballeure, à son vélo à pignon fixe ou à la couleures de sona absence de voiture ou de tracteurice.

En outre, même vêtuz d'un complet gris - cravatte nacarat, alquanz "en beige" (homme comme femme) restait beigeable à sa couleur.

Les couleurs ontologiques s'exprimaient ainsi très distinctement, sans avoir à se situer sur teu_telle longueur d'onde.

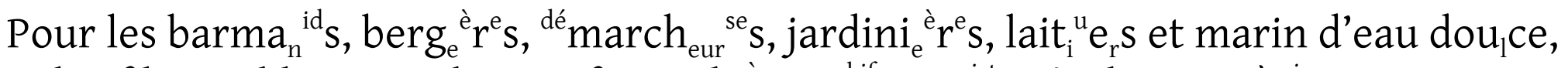

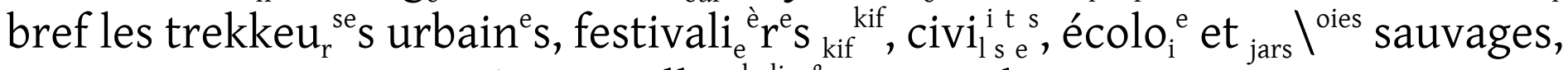
qu'on appelle "ladies\& gen $_{t}$ s en brun",

les marques ad hoc proposèrent donc tout ${ }_{\mathrm{s}}^{\mathrm{e}}$ sauf des chaussures brunes :

Parkas GEOX terre de Sienne en Gore-Tex, vestes baies et fraise Mammut et chaussures quand même mais châtain The North Face et fauves Jack Wolfskin.

En fin de compte, peu importe l'accessoire ou sa couleur,

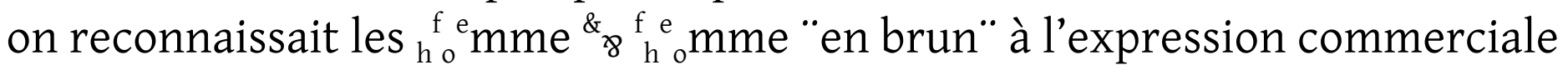
de leur orientation politique vert'libérale, voire verts pour les plus invariables. C'était comme si l'idéologie s'alignait harmonieusement au choix des marques.

C'est alors que les Bobos à Paris, les BuBö à Vienne, les babas à Rome et tou ${ }_{\mathrm{s}}^{\text {te }}$ les intellectuel ${ }^{\text {les }}$ malàdroite, qui n'achètent qu'en seconde main et n'avaient donc jusqu'à présent pas été distinguée de la masse des "transparent" qui n'achètent non plus rien de nouveau ni de visible ni n'osent le porter, furent alors en mesure d'exprimer leurs opinions sur les beles ${ }_{-1}^{\text {les }} \mathrm{ett}$ es, les loupvs et les renarts, en continuant simplement de boire du caviar et de manger du Champagne en Cüpli. ${ }_{1}^{\mathrm{E}} \mathrm{l}^{\mathrm{le}} \mathrm{s}$ étaient enfin entrées dans la ronde, et n'avaient pourtant ni chaussures neuves - si ce n'est de pures trouvailles ni vélo actuel - mais bicyclette pas moins rarissime pour autant. 
À l'orée du socialisme "brun", les milices de l'asservissement civil, voulant se démarquer dans l'objection de conscience antimode, ne portèrent alors que les ${ }^{\mathrm{TM}} \mathrm{shirts}$ du service et/ou de la protection civile . Trop de marques, trop de libéralisme pour ces vibrant ${ }^{\mathrm{e}} \mathrm{s}$, ces méditant ${ }^{\mathrm{e}} \mathrm{s}$...

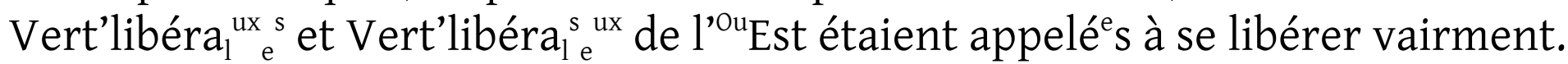
Ils ${ }_{\text {Illes }}$ scandaient ainsi : « Nous ne sommes pas des consommat non! nonne! !

La censure et la prohibition devint vite le fond de commerce du temple anar.

René ${ }^{\mathrm{s}}$ et renégat ${ }^{\mathrm{e}} \mathrm{s}$, apôtres et apostat ${ }^{\mathrm{e}} \mathrm{s}$ disparurent vivant ${ }^{\mathrm{e}} \mathrm{s}$ dans le sans-corps électoral réac'/révolu'/rebel'.

En imitant la FAQ originelle «acheter ou bien ne pas acheter?» avait été résolu le dilemme ontologique « être ou plutôt ne pas être? ».

La sinistre convention des sans-culottes et des dextres gens de robe initia donc l'étape cruciale : le noir dans l'âme et non dans l'épiphanie terrestre.

Le groupement négateur "en noir" n'a pas encore / plus de corps, ni moins de sexe / genre / sexualité.

L'invariable (sans-)chair n'a pas non plus de noir pour (ça) vêtir ; le noir n'est pas dans les fibres textiles, il est l'absence d'attention portée au look.

On se mit dès lors à confondre apparence et intentions. Noir le personnel acoustique des salles de concert, noir le collectif de ramonage, nègre l'internationale de la pègre en leurs unités membrues. Comment distinguer le fil rouge du fil blanc dans tant de noir?

Le premier incident en ce genre de discrimination eut lieu lors d'un concert bourgeois de musique de chambre.

Alors que toz les musiciegnz, tout de noir vestuz, arboraient des racines colorées, le service de tournage de page voulant s'éclipser discrètement avait acheté pour l'occasion des Dr. Martens noir "noir". 
Priz un instant dans un virage bancal, læ flûtiste partit en trille et l'on aurait pu croire ce grincement prévu, si ce n'est orchestré, d'autant plus que l'interprète portait des baskets bleu-rouge à la Batman ; personne n'attendait vraiment d'èl une exécution parfaite, "beige" ni "dorée". Mais à cet instant, tout le public remarqua le regard traversier envers læ flûtiste que lança spontanément le service "en noir" derrière la queue du piano.

La bécasse de flûtiste croula de honte à cause de cette couille, si bien que l'on dût interrompre le concert en raison de cet évènement.

Chascunz n'avaient qu'une monoscopie de l'incident :

si cest pauvre flûtiste avait raté son solo de façon si remarquée, c'est parce que cèt salaudə, cèl espèce de groß salopə de service de chambre, l'avait regardé comme unə anarchiste // comme par anarchisme.

Ça descendit manifester dans les rues. Le terrorisme s'en prit à la Boîte Noire, lançant des pavés contre des religieuses standards et d'autres têtes de Turques.

Alors, invisible mais rose, la Licorne égaya un peu cette ambiance trou noir avec ses parades « Mariage pour [n|v]ous! » anti-euphorie-nation/langue/religion.

La communauté était multicompositée de tout le spectre RVB de l'arc-en-ciel : des femmes comme vous et (des hommes comme) moi, un Chaperon Rouge, un Cendrillon et princesse $\{s\}$ pour danser avec lui, des passivs et des activs, six filles cis* et yn personne flipflop ${ }^{\mathrm{TM}}$, des romanescos quelque homo erectus, tous les varia d'un milieu ménopausé BDSM enceint $[\mathrm{F} \mid \mathrm{H}] \mathrm{S}[\mathrm{F} \mid \mathrm{H}]$ diva barbu $T^{*} t W T F$.

Cellesefeux "en violet" maîtrisaient depuis bel lurette les épicénités homophones de l'écriture non outante. Celleseßeux dürfaient aussi abuser des formes passives afin qu'il ne soit pas su la dysphorie sexe/genre/orientation de la compagne avec laquelle on disait partir en vacances à la campagne « avec partenaire ». Le kilt violet n'était le fantasque que des gens de robe et alcunz ne put vendre nennil à l'inversion Queer. 
Leur engagement pour une nouvelle astérixade les motivait désormais :

l'écriture non quantitative - contre/pour le pluri*el -

qui permettrait de sensibiliser la populace généralisée qu'il y a une différance

entre un bel amour et des belles amours

et qu'il est singulexiste de toujours renforcer le cliché de la monogamie en limitant le nombre de tantes que l'on peut monter au Jardin d'Éden.

Et de scander donc «Espace s pour $\left[{ }_{n \mid v}||^{t}\right]$ ou $\left[{ }^{\text {te }} \mid s\right]$ ! » avec typographie insécable, pour dire que la question n'est pas de savoir si [les |l']espace[s] est d'un genre binaire

mais de se demander si un nombre bigenre d'espaces permettrait la polyamorie.

Cet' étape $\mathrm{un}_{\mathrm{e}}$ tantinet $_{\mathrm{te}}$ théorique servit à rappeler à ces animalux heureuz et doulz d'hétérosexistes conser ${ }^{f}$ vasteurs ${ }_{\text {femme }}^{(}$( homme, "en $b_{1} e_{u}{ }^{\text {ige.". }}$

qu'il fallu't parfois affronter la nuict intérieure au lieu de regarder par la fenestre et accuser le $e_{s}$ vetement ${ }_{s}$ "en noir" d'abriter le Costé Obscur.

Les sept genres violette ${ }_{G \text { T Q }}^{\mathrm{L}}$ B I étaient là (et lè), parmis nous, et ce n'était ni leurs Nike rouges, ni lor sac à main brun, ni leurs habites cool, leurs parfumes, shampooignes, savonnes qui leur donnaient un genre

(ni une nombre), mais uniquement leur quintessence de violette.

On était "violet" profondément, de l'intérieur,

et ce n'était pas le grand manteau noir qui faisait l'exhibitionniste.

On pouvait même être simultanément "en violet" et "en une autre couleur", comme le démontrèrent les associations politiques vert'PINK CROss et vert'LOS menées par des divas "en brun", une Wurst et une cantatrice chauve.

Mais c'est surtout en appliquant cette observation à une sous-espèce de baleines et de cougars, de baleines et de cougars, socialistes saumon fumé irlandais, socialistes salmonelle fumée irlandaises, petits bobos bénins que le grand public droite cervelas et cerveau droit réalisa que ces petites bobettes peu malignes 
les "gens en rouge"

n'étaient ni brun ${ }_{\mathrm{s}}^{\mathrm{s}}$ comme les bobos graves ni ne portaient des baskettes rouges. Ces bonshommes de neige aux grands airs bonhommes,

Ces bonnes femmes de glace portaient certainement le rouge aussi bien que l'embonpoint et jouissaient certainement d'autant d'extravagance que Michael Jackson ou Madonna, Michaela Jacksdóttir ou Mondon, mais n'étaient "en rouge" que dans la manière dont les autres

- Terre des hommes - Gaïa des femelles parlaient d'eux. parlaient d'elles.

Ils étaient camelots, préfets, prud'hommes, homo ludens. Bourreaux des cœurs parfois. Bourrus aussi.
Elles étaient sapeuses-pompières chocolatières, clowns, chefs, homo demens. Bourrelles des cervelles souvent. Bourriques *holà !

On pouvait être "en rouge" par obésité, maladresse, laideur ou extravagance, comme on aurait pu pour cela être "en rose", "en beige", "en gris" resp. "en vert", on portait parfois du rouge, même aux pieds, mais surtout partout ailleurs, comme le Père Noël et ses comme la Mère Noëlle et ses déviations standards ou écarts-types, déviations standards ou écartent-filles, mais on était avant tout un particulier "en rouge" de cas en cas, par définition, par sémiologie typographique, sans autodétermination. La Grande Famille "en violet" connaissait sa généalogie, tandis que l'on pouvait porter en@sur soi les lettres écarlates et tou tefoi $\mathrm{s}$ l'ignorer.

Le commerce de la chaussure et des produites dérivéєs n'y étaient pour plus rien=ne.

Tant*e s'était passé*e dans la sociétéE depuis qu'on avait commencé à repérer plusieur $^{2,71828}$ s analogies entre les couleurs et les personnes humaines. 
On approchait alors de la déconstruction, de l'oubli, de la fuite.

Comment cella se produisie? C'est pas sorcièr !

Les chômeureuses malheureuxeuses et les usagérées de droguées intraveineureuses

rouges comme des verres de bœufs, jaunes comme des jaunisses,

cirrhotées-osées, gris de cuites comme le vert ivre du cuivre,

infectées par des godasses qui se transmettent d'un candida à l'autrèque, claudiquant entre une Converse à son pied gauche et une adidas maladroite,

les sidaliennes et les séropositivistes teulx qu'on les imaginères d'Épinales, se mirent simplement à porter à leur-s tour-s les fameuxes soulières colorices.

\section{Rappelées-vouses;}

les tennisses rouges étaient la marque des "gens en rose" ou moitié d"'orange", mais lorsque portéz par unsE alcoelhotabagićque, l'hypothais(e) été-e fauxse ;

les dorures éclaoncles de

\section{le|la Lune}

l• Soleil•le

auraient $\mathrm{du}_{\mathrm{e}}^{\mathrm{s}}$ leur cou'ter un salère d'acteur...ine s'illex étaient "en dorö", mais vue que le manteau_la mantelle en question ${ }^{\circ}$ ne avèz éetée trouvé dans un/e poubel/poubeau, cellela ne pouvaitte être qu'un humain "en jaune".

L'extrême décadence peut-elle encore porter un signifié comme chaque semelle possède sa propre trace?

Les " $\square$ en blanc" sont la dé-personnalisation même. Les innocent $\square$ que l'on retrouve perdu $\square$ dans les toilettes des $\square$, n'ont pas encore de psychisme, pas encore de conscience ou peut-être déjà plus. se contentent d'exister, mais portent cependant des chaussures

- parfois blanches, mais ce serait là un hasard trop pur des chaussures hautes en couleur certainement, et des habits de hipster - rien de plus moche et pourtant, à Baselle au bord de sa Rhin·e, $\square$ restent "blanc" Toutes LES ANnées. 